\title{
Direct numerical simulation of turbulent intermediate Froude number fountain flow
}

\author{
N. Williamson ${ }^{1} \quad$ S. W. Armfield ${ }^{2} \quad$ W. Lin ${ }^{3}$
}

(Received 12 August 2008; revised 28 August 2008)

\begin{abstract}
Fountains are an important flow in many industrial and geophysical problems but the understanding of this flow at low Froude numbers is limited. In this study we use direct numerical simulation to examine two regimes of turbulent fountain flow, weak flow at $\mathrm{Fr}=2.2$ and very weak flow at $\mathrm{Fr}=0.45$. At $\mathrm{Fr}=0.45$ buoyancy stabilises the flow and there is little entrainment of ambient fluid. Kelvin-Helmholtz instabilities are generated at the interface between the upwelling flow and the ambient fluid. At $\mathrm{Fr}=2.2$ the shear interaction between the rising and falling flow streams becomes important as it drives both large and small scale vortex structures. Large scale structures draw ambient fluid into the fountain while small scale structures exchange mass between the upflow and downflow. Periodic ejection of mass from the top of the fountain is shown to be an important dynamic in the flow. The results provided here can assist in analytical model development.
\end{abstract}

See http://anziamj.austms.org.au/ojs/index.php/ANZIAMJ/article/view/ 1419 for this article, (c) Austral. Mathematical Soc. 2008. Published August 29, 2008. ISSN 1446-8735 


\section{Contents}

1 Introduction

C17

2 Numerical formulation

C19

3 Results

C21

4 Conclusions

C27

References

C27

\section{Introduction}

Fountains occur when a fluid is injected into another fluid of differing density where the buoyancy force opposes the initial momentum at the source. The injected fluid flow penetrates a distance into the ambient fluid before stagnating and then flowing back around the rising column in a dense plume. This flow structure occurs in many important industrial and geophysical flows. Previous studies have included fountain flows related to the flow of lava in magma chambers [1], air-conditioning and heating in large buildings [2], replenishment of solar ponds [3], pyroclastic clouds [4] and evaporating atmospheric plumes [5]. The characteristics of turbulent fountain flow depend primarily on the Froude number for the flow, defined for round fountains as

$$
\operatorname{Fr}_{0}=\left(\frac{M_{0} U_{0}}{F_{0} R}\right)^{1 / 2}
$$

where $R$ is the radius of the source, and $M_{0}$ and $F_{0}$ are the momentum flux and buoyancy flux at the source respectively. They are defined as $M_{0}=$ $\int_{0}^{R} 2 \pi r \bar{U}^{2} d r$ and $F_{0}=\int_{0}^{R} 2 \pi \sigma r \bar{U} d r$ in which $\bar{U}$ is the local time averaged axial velocity, and $\sigma=g\left(\rho_{0}-\rho_{f}\right) / \rho_{f}$ is the reduced gravity between the 
fountain source and the ambient fluid. The subscript 0 indicates a quantity at the fountain source and $f$ indicates a property of the ambient fluid. The characteristic velocity $U_{0}=Q_{0} / \pi R^{2}$, where $Q_{0}$ is the volume flow rate at the fountain source.

At high Froude numbers, fountain flow becomes turbulent close to the source and there is strong mixing and entrainment between the rising and falling flow regions and the ambient fluid $[5,6,7]$. This flow regime has been extensively studied $[2,5,6,7,8]$ and recently reviewed by Williamson et al. [9]. The consensus of these works is that for high Froude number turbulent round fountains the maximum penetration depth $Z_{m}$ scales as $Z_{m} / R=C F r_{0}$, where $C$, a constant of proportionality, is independent of Reynolds number $R e=U_{0} R / v_{0}$. The scaling relations for low Froude number behavior differ from the high Froude number result. At low Froude number $\mathrm{Fr}_{0} \lesssim 1$, the buoyancy forces are dominant and the flow penetrates a relatively small distance into the ambient fluid with very little mixing or entrainment $[3,8,10,11]$.

This change in the flow behavior reflected in the scaling relations is an important problem. A number of studies worked to define limits over which subranges of behavior exist in turbulent round fountains [3, 8, 10, 12]. Recently Kaye and Hunt [8] suggested a three regime classification of fountain behavior, labeling them 'forced' for $\mathrm{Fr}_{0}>3$, 'weak' for $1<\mathrm{Fr}_{0}<3$ and 'very weak' for $\mathrm{Fr}_{0}<1$. The primary differentiator between the regimes is the relative influence of entrainment and buoyancy on the mean flow. Using an analytical model based on Morton, Taylor and Turner's entrainment model [13] and assuming entrainment in high Froude number fountains is similar to that in non-buoyant jets, they found the well established result of $Z_{m} / R \sim F_{0}$. For weak flow Kaye and Hunt supposed that the influence of entrainment is small compared with the direct buoyancy forces so it can be neglected in the model. This approach yielded $Z_{m} / R \sim \mathrm{Fr}_{0}^{2}$, a result which is fairly well supported by experimental results [3, 8]. For very weak fountains Kaye and Hunt related the radial outflow of fountain fluid from the nozzle 
to flow over a weir. Using this analogy they found $Z_{m} / R \sim \mathrm{Fr}_{0}^{2 / 3}$, the same Froude number scaling obtained by Lin and Armfield [10]. Both the analytical model and the experimental results show that the transitions between the flow behaviors occur at $\mathrm{Fr}_{0} \approx 1$ and $\mathrm{Fr}_{0} \approx 3$.

The mechanisms governing the flow at these low Froude numbers have not been well described, so there is not a strong basis for the development of any analytical model. The objective of the present study is to provide additional insight into the energy and mass transfer processes that occur within low Froude number fountains. We use direct numerical simulation of fully turbulent fountain flow at Froude numbers of 0.45 and 2.2 to investigate the dominant flow mechanisms associated with each flow regime.

Our main findings are that for very weak fountain flow all mixing and entrainment is confined to a thin interface region between the upflow and the ambient and the intrusion region. The lack of shear turbulence production and the action of buoyancy stabilises the flow. For weak fountains a region of strong shear between the upflow and downflow drives large scale engulfment of ambient/downflow fluid into the mixing region and small scale mixing between the upflow and downflow.

\section{Numerical formulation}

We use direct numerical simulation (DNS) to solve the Navier-Stokes equations for incompressible flow and employ the Boussinesq approximation. The non-dimensionalised continuity, momentum and scalar transport equations are

$$
\begin{aligned}
& \frac{\partial u_{i}}{\partial x_{i}}=0, \\
& \frac{\partial u_{i}}{\partial t}+\frac{\partial\left(u_{i} u_{j}\right)}{\partial x_{j}}=-\frac{\partial p}{\partial x_{i}}+\frac{1}{\operatorname{Re}} \frac{\partial^{2} u_{i}}{\partial x_{j} \partial x_{j}}+\frac{\phi}{F_{0}^{2}},
\end{aligned}
$$




$$
\frac{\partial \phi}{\partial t}+\frac{\partial\left(u_{j} \phi\right)}{\partial x_{j}}=\frac{1}{\operatorname{Re} \operatorname{Pr}} \frac{\partial^{2} \phi}{\partial x_{j} \partial x_{j}},
$$

where $\operatorname{Pr}$ is the Prandtl number and the non-dimensional temperature $\phi=$ $\left(T-T_{f}\right) /\left(T_{f}-T_{0}\right)$. Velocity, $u_{i}$, is non-dimensionalised by $U_{0}$ and length by $R$. The discretised governing equations were solved in finite volume form on a non-staggered grid. The spatial derivatives were discretised using second order central finite differences. The ULTRA-flux limiter was applied to the scalar advective term [14]. The advective terms were advanced in time using the second order Adams-Bashforth scheme while the viscous terms were advanced using the Crank-Nicolson scheme. The system of equations was solved with the BICGSTAB solver with the Jacobi pre-conditioner.

The computational domain is a rectangular box in which the top wall is an open boundary and the sides are adiabatic no-slip walls. The bottom boundary is also no-slip/adiabatic, except for the fountain source located in the centre. The inflow at the fountain source is described by an unsteady boundary condition derived from a separate DNS simulation of turbulent pipe flow at $R e=3350$. The velocity field was recorded across a single crosssection of the pipe during run time. The recorded flow is played back in the present fountain simulation at the fountain source.

Simulations were conducted at two Froude numbers, $\mathrm{Fr}_{0}=0.45$ and $\mathrm{Fr}_{0}=$ 2.2 corresponding to the weak and very weak regimes of Kaye and Hunt [8]. The Reynolds number was $\operatorname{Re}=3350$, and Prandtl number $\operatorname{Pr}=7.0$. A regular cartesian grid was used. For the $\mathrm{Fr}_{0}=0.45$ simulation, the domain was $\mathrm{L}_{x}=15 R, \mathrm{~L}_{y}=15 \mathrm{R}$ and $\mathrm{L}_{z}=5 \mathrm{R}$ where the $z$-axis is aligned with the source inflow. The grid is uniform up to $1.2 R$ from the center of the source in the $x$ and $y$ directions, thereafter it is stretched at $6 \%$. In the vertical direction the grid is uniform up to $0.65 \mathrm{R}$ and thereafter is stretched at $12 \%$. A total of 200 nodes were located across the uniform mesh region in the $x y$-plane and a total of 58 nodes in the vertical direction. Across the entire domain the resolution was $320 \times 320 \times 100$ nodes in the $x, y$ and $z$ directions. This resolves down to approximately 2.2 times the Kolmogorov length scale 
in the fountain core region. The resolution of the domain outside this region is less important in this study. In the $\operatorname{Fr}_{0}=2.0$ simulation the domain was $\mathrm{L}_{x}=40 \mathrm{R}, \mathrm{L} y=40 \mathrm{R}$ and $\mathrm{L}_{z}=30 \mathrm{R}$ with $210 \times 210 \times 180$ nodes in the $x, y$ and $z$ directions, with the uniform grid up to $1.2 \mathrm{R}$ from the center of the source in the horizontal plane and $4.0 \mathrm{R}$ in the vertical direction, thereafter with the same stretching as above. The resolution of the simulation is approximately six times the Kolmogorov length scale in the fountain core region. The CFL number for the flow was limited to between $0.15-3.0$ in both simulations. The simulations were run a total of $40 \mathrm{R} / \mathrm{U}_{0}$ time steps for $\mathrm{Fr}_{0}=2.2$ and $15 \mathrm{R} / \mathrm{U}_{0}$ for $\mathrm{Fr}_{0}=0.45$, with statistics collected after fully developed flow where the downflow is fully established.

\section{$3 \quad$ Results}

This section describes both the transient features and flow structures of the two fountains and then examines the internal mass transfer processes in more detail with the turbulent flow statistics. The structure of the $\mathrm{Fr}=0.45 \mathrm{flow}$ is shown in Figure 1, where a contour plot of temperature at $t=3 R / U_{0}$ is given, overlaid with velocity vectors, to illustrate the flow field. In this very weak fountain simulation, the flow penetrates a short distance into the ambient fluid before the cap region at the top of the fountain is formed and the fluid spreads radially outwards forming an intrusion in the ambient fluid.

The cap region remains very steady with little oscillation in fountain height. The interface between the outflowing fountain fluid and ambient fluid is very sharp with no entrainment of ambient fluid into the fountain core. At the interface of the fountain with the ambient fluid, Kelvin-Helmholtz vortices form and flow into the intrusion region where they break up into small scale structures. From the velocity vectors it is evident that there is strong radial acceleration of the fluid through the cap region into the intrusion. Apart from the Kelvin-Helmholtz structures in the interface, the 


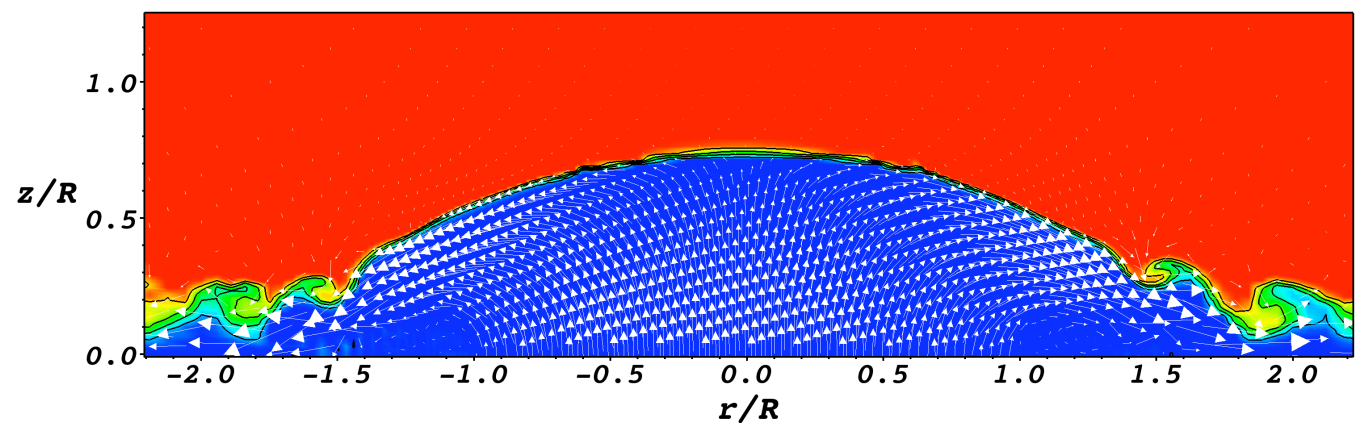

Figure 1: Contours of temperature with velocity vectors for $\mathrm{Fr}_{0}=0.45$ at $\mathrm{t}=3 \mathrm{R} / \mathrm{U}_{0}$. Velocity vectors are scaled with magnitude.

flow is quite axisymmetric. The turbulent inflow is quickly stabilised and vortex structures advected into the domain from the turbulent boundary condition are advected out into the intrusion.

The turbulent statistics for the $\operatorname{Fr}_{0}=0.45$ simulation are given in Figure 2. The temperature variance $\left\langle\phi^{\prime} \phi^{\prime}\right\rangle$, the temperature velocity correlations $\left\langle\phi^{\prime} u_{a}\right\rangle$ and $\left\langle\phi^{\prime} u_{r}\right\rangle$ and the shear stress all peak at the same location in the interface region and extend into the intrusion layer. The mixing is entirely contained in the very thin interface region through which the KelvinHelmholtz instabilities pass and in the intrusion region. The buoyancy term in the kinetic energy equation, not shown here, is dissipative in this region; that is, it acts to suppress fluctuations and is of comparable magnitude to the other terms in the kinetic energy balance. The fluctuations from the turbulent pipe inflow are small compared with those in the intrusion region and do not register on the contour plots in Figure 2.

At $\mathrm{Fr}_{0}=2.2$, the flow penetrates a greater distance into the ambient domain. There are clearly defined boundaries between the upflow, downflow and ambient fluid. At the interface between these streams mixing occurs and fluid and momentum is transferred. 

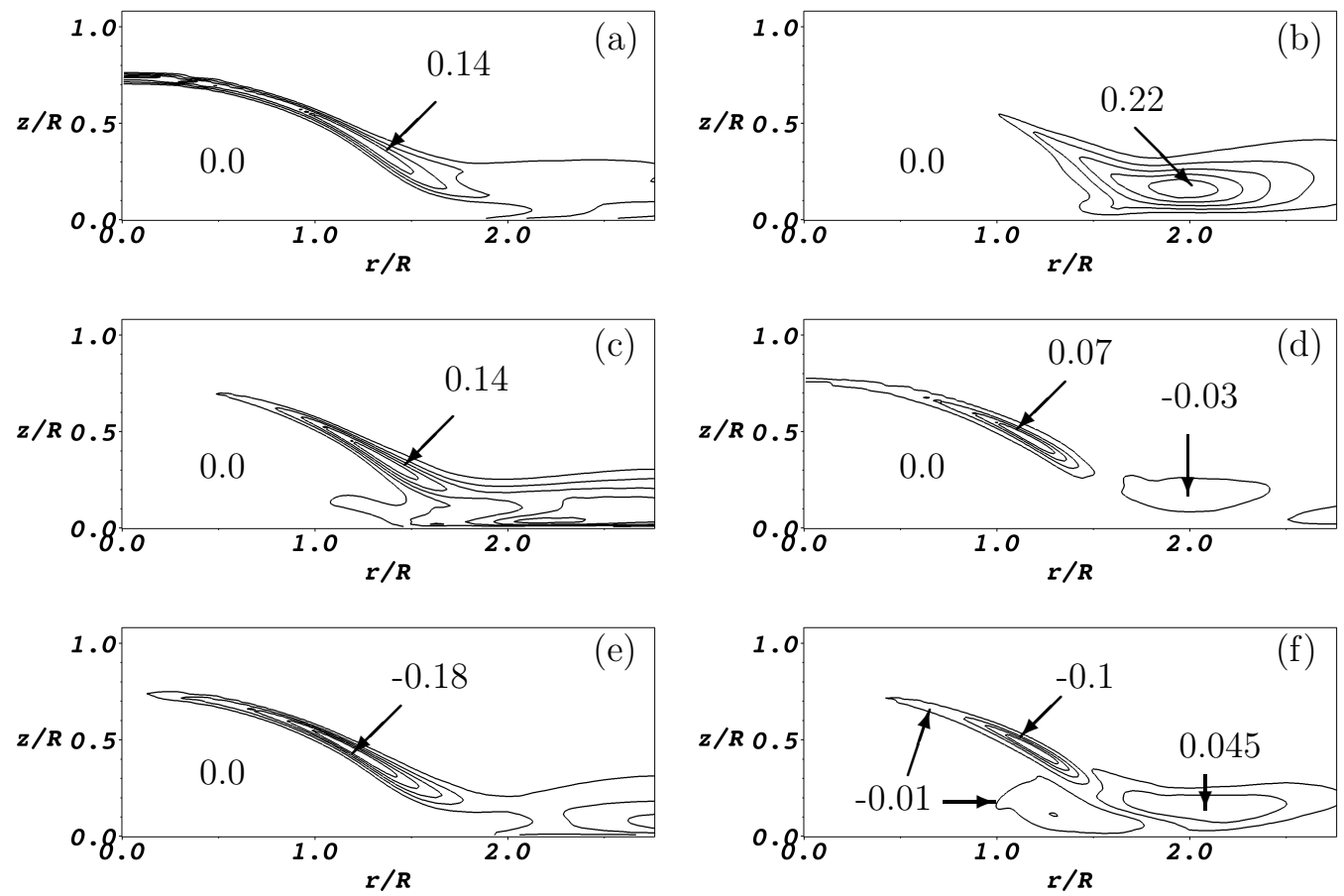

Figure 2: Contours of flow variables at Froude number $\mathrm{Fr}_{0}=0.45$; (a) $\left\langle\phi^{\prime} \phi^{\prime}\right\rangle$, (b) $\left\langle u_{a} u_{a}\right\rangle$, (c) $\left\langle u_{r} u_{r}\right\rangle$, (d) $\left\langle\phi^{\prime} u_{a}\right\rangle$, (e) $\left\langle\phi^{\prime} u_{r}\right\rangle$, (f) $\left\langle u_{r} u_{a}\right\rangle$. 
In Figure 3 a series of images illustrate the $\mathrm{Fr}_{\mathcal{O}}=2.2$ flow development. Several important unsteady flow features are evident. Fountain fluid accumulates in the cap region and is shed periodically as a cohesive lump causing the fountain height to oscillate. This shedding motion is important for entrainment. In Figure 3 the lump of mass is labelled 'A'. The progression of this collapse is shown through the figures with time. In Figure 3(a) the lump is forming which is accompanied by a strong region of inflow into the fountain core from the downflow and the ambient fluid, as indicated by 'B'. By Figure 3(b) the ambient fluid has been drawn into the core region and the lump is moving into the downflow. When the lump reaches the base of the fountain the strength of the downflow increases the shear there and drives a strong vortex structure at the base of the fountain labeled ' $\mathrm{C}$ ' in Figures 3(c) and (d). This vortex draws the descending flow back into the mixing region between the upflow and downflow. Thus two types of large scale structures have been observed, those driven by collapse and those driven by the shear in the downflow. Small scale structures in the mixing region are also highlighted in the figures with the label ' $\mathrm{D}$ '. The contours show the entrainment and mixing can extend into the fountain core region, in contrast to the very weak fountain flow.

The turbulent fluctuations shown in Figure 4 show the presence of two important zones, the inner shear region between the upflow and downflow where $\left\langle u_{r} u_{a}\right\rangle$ peaks and a second outer region between the downflow and the ambient. The temperature fluctuations are strongest in the cap region and in the downflow where large long time scale fluctuations occur. The temperature fluctuations in the inner mixing region are much smaller as the fluid here is already well mixed. The normal stresses peak in the shear region, which is also the region of peak kinetic energy production. 

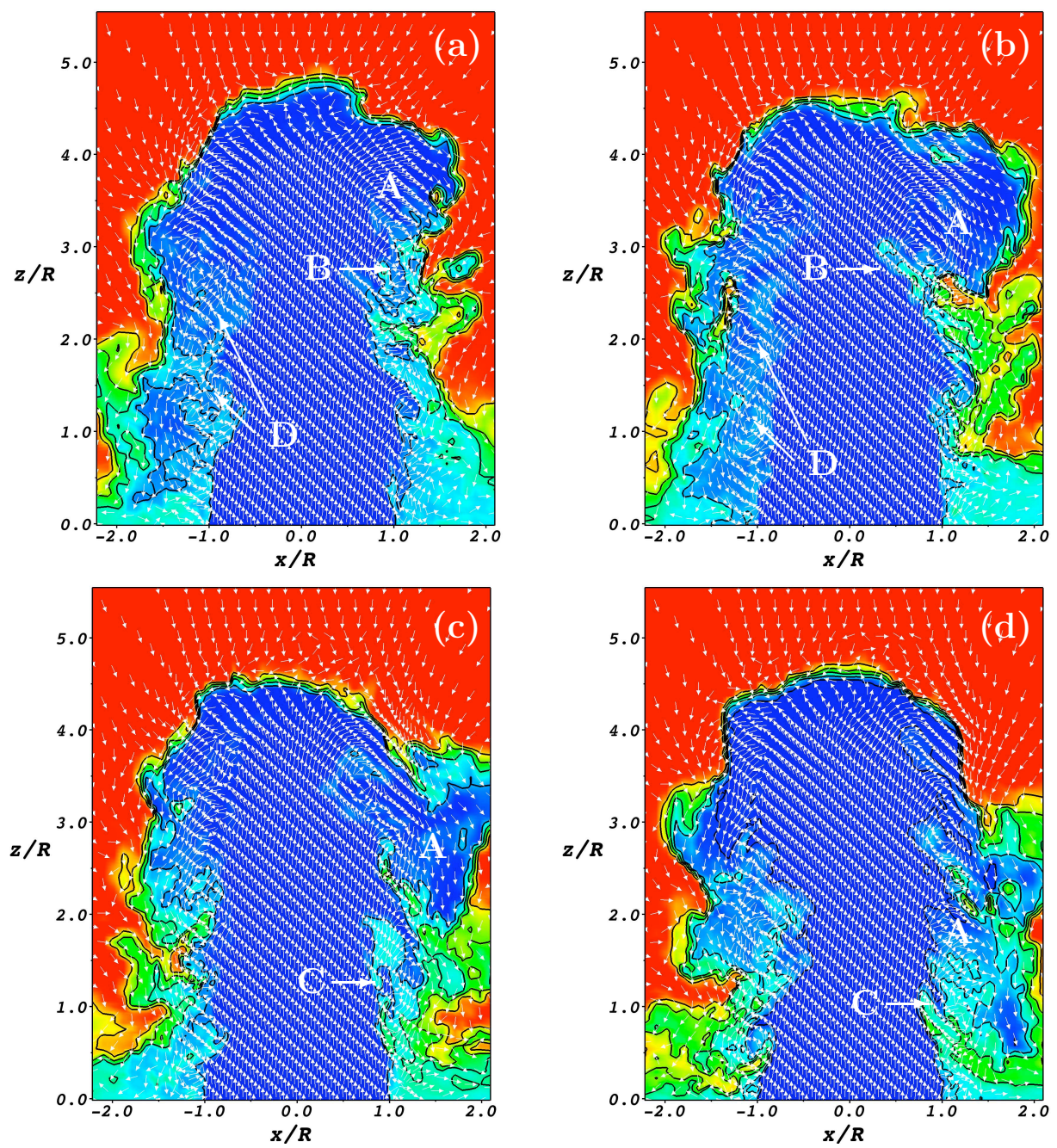

FIGURE 3: Contours of temperature with unscaled velocity vectors for Froude numbers $\mathrm{Fr}_{0}=2.2$ at time $(\mathrm{a}) \mathrm{t}=30.6 \mathrm{R} / \mathrm{U}_{0},(\mathrm{~b})+1.8 \mathrm{R} / \mathrm{U}_{0},(\mathrm{c})+3.6 \mathrm{R} / \mathrm{U}_{0}$, (d) $+5.4 \mathrm{R} / \mathrm{U}_{0}$. 

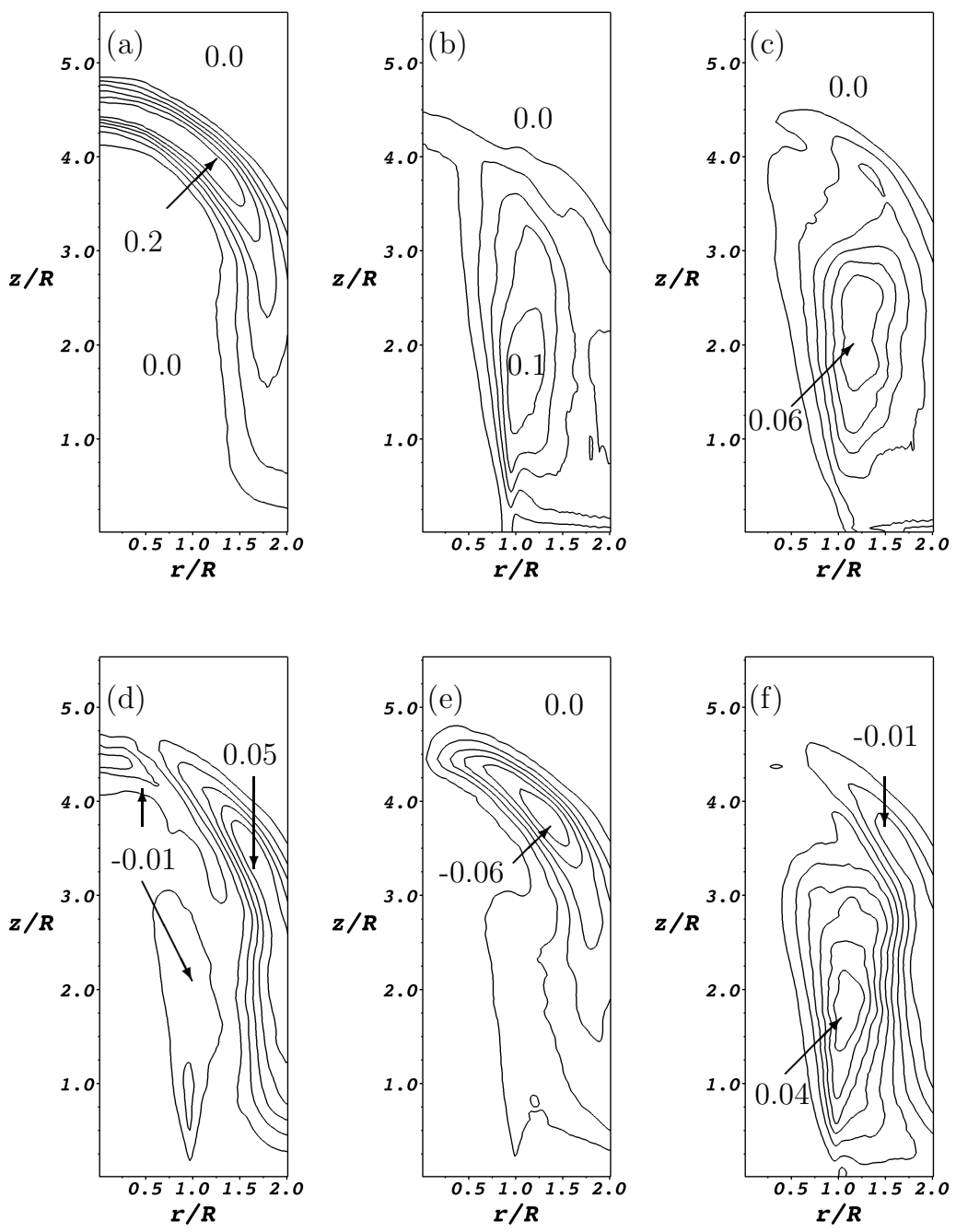

Figure 4: Contours of flow variables at $\operatorname{Fr}_{0}=2.2$ : (a) $\left\langle\phi^{\prime} \phi^{\prime}\right\rangle$, (b) $\left\langle u_{a} u_{a}\right\rangle$, (c) $\left\langle u_{r} u_{r}\right\rangle$, (d) $\left\langle\phi^{\prime} u_{a}\right\rangle$, (e) $\left\langle\phi^{\prime} u_{r}\right\rangle$, (f) $\left\langle u_{r} u_{a}\right\rangle$. 


\section{Conclusions}

For very weak fountain flow, buoyancy stabilises the flow and all mixing and entrainment is confined to a thin interface region and the intrusion region. For weak fountains, we found that the increased penetration height introduces a region of strong shear between the upflow and downflow. This drives two mechanisms of entrainment, large scale engulfment of ambient/downflow fluid into the mixing region and small scale mixing between the upflow and downflow. Fluid is ejected from the top of the fountain periodically as large rotating structures. These structures draw ambient fluid into the upflow and significantly influence the flow dynamics.

Acknowledgements The authors acknowledge the support of the Australian Research Council.

\section{References}

[1] I. H. Campbell and J. S. Turner. Fountains in magma chambers. J. Petrol., 30:885-923, 1989. http://petrology.oxfordjournals. org/cgi/content/abstract/30/4/885 C17

[2] W. D. Baines, J. S. Turner, and I. H. Campbell. Turbulent fountains in an open chamber. J. Fluid Mech., 212:557-592, 1990. doi:10.1017/S0022112090002099. C17, C18

[3] W. Lin and S. W. Armfield. Direct simulation of weak axisymmetric fountains in a homogeneous fluid. J. Fluid Mech., 403:67-88, 2000. doi:10.1017/S0022112099006953. C17, C18 
[4] E. Kaminski, S. Tait, and G. Carazzo. Turbulent entrainment in jets with arbitrary buoyancy. J. Fluid Mech., 526:361-376, 2005. doi:10.1017/S0022112004003209. C17

[5] J. S. Turner. Jets and plumes with negative or reversing buoyancy. J. Fluid Mech., 26:779-792, 1966. doi:10.1017/S0022112066001526. C17, C18

[6] T. Mizushina, F. Ogino, H. Takeuchi, and H. Ikawa. An experimental study of vertical turbulent jet with negative buoyancy. Wärme-und Stoffübertragung, 16:15-21, 1982. doi:10.1007/BF01322802. C18

[7] R. W. Cresswell and R. T. Szczepura. Experimental investigation into a turbulent jet with negative buoyancy. Phys. Fluids A, 5:2865-2878, 1993. doi: $10.1063 / 1.858749$. C18

[8] N. B. Kaye and G. R. Hunt. Weak fountains. J. Fluid Mech., 558:319-328, 2006. doi:10.1017/S0022112006000383. C18, C20

[9] N. Williamson, N. Srinarayana, S. W. Armfield, G. D. McBain, and W. Lin. Low-reynolds-number fountain behaviour. J. Fluid Mech., 608:297-317, 2008. doi:10.1017/S0022112008002310. C18

[10] W. Lin and S. W. Armfield. Very weak axisymmetric fountains in a homogeneous fluid. Numer. Heat Transfer A, 38:377-396, 2000. doi:10.1080/104077800750022520. C18, C19

[11] P. D. Friedman, V. D. Vadokoot, W. J. Meyer, and S. Carey. Instability threshold of a negatively buoyant fountain. Exp. Fluids, 42:751-759, 2007. doi:10.1007/s00348-007-0283-5. C18

[12] H. Zhang and R. E. Baddour. Maximum penetration of vertical round dense jets at small and large Froude numbers. J. Hydraulic Eng., 124:550-553, 1998. doi:10.1061/(ASCE)0733-9429(1998)124:5(550). C18 
[13] B. R. Morton, G. I. Taylor, and J. S. Turner. Turbulent gravitational convection from maintained and instantaneous sources. Proc. R. Soc. Lond., 234:1-23, 1956. http://www. jstor.org/stable/99936. C18

[14] B. P. Leonard and S. Mokhtari. Beyond first-order upwinding: the ultra-sharp alternative for non-oscillatory steady state simulation of convection. Int. J. Numer. Meth. Engng, 30:729-766, 1990. doi:10.1002/nme.1620300412. C20 


\section{Author addresses}

1. N. Williamson, School of Aerospace, Mechanical and Mechatronic Engineering, University of Sydney, Australia. mailto:n.williamson@usyd.edu.au

2. S. W. Armfield, School of Aerospace, Mechanical and Mechatronic Engineering, University of Sydney, Australia.

3. W. Lin, School of Engineering, James Cook University, AUstralia. 